\title{
Esophageal Reconstruction with Gastric Pull-up in a Premature Infant with Type B Esophageal Atresia
}

\author{
Young Mi Han, Narae Lee, Shin Yun Byun, Soo-Hong Kim*, Yong-Hoon Cho*, and Hae-Young Kim* \\ Division of Neonatology, Department of Pediatrics, Pusan National University Children's Hospital, Pusan National University School \\ of Medicine, Yangsan, Korea \\ Division of Pediatric Surgery, Department of Surgery*, Pusan National University Yangsan Hospital, Pusan National University School \\ of Medicine, Yangsan, Korea
}

\section{ABSTRACT}

Esophageal atresia (EA) with proximal tracheoesophageal fistula (TEF; gross type B) is a rare defect. Although most patients have long-gap EA, there are still no established surgical guidelines. A premature male infant with symmetric intrauterine growth retardation (birth weight, 1,616 g) was born at 35 weeks and 5 days of gestation. The initial diagnosis was pure EA (gross type A) based on failure to pass an orogastric tube and the absence of stomach gas. A "feed and grow" approach was implemented, with gastrostomy performed on postnatal day 2. A fistula was detected during bronchoscopy for recurrent pneumonia; thus, we confirmed type B EA and performed TEF excision and cervical end esophagostomy. As the infant's stomach volume was insufficient for bolus feeding after reaching a body weight of $2.5 \mathrm{~kg}$, continuous tube feeding was provided through a gastrojejunal tube. On the basis of these findings, esophageal reconstruction with gastric pull-up was performed on postnatal day 141 (infant weight, $4.7 \mathrm{~kg}$ ), and he was discharged 21 days postoperatively. At 12 months after birth, there was no catch-up growth; however, he is currently receiving a baby food diet without any complications. In patients with EA, bronchoscopy is useful for confirming TEF, whereas for those with long-gap EA with a small stomach volume, esophageal reconstruction with gastric pull-up after continuous feeding through a gastrojejunal tube is worth considering.

Key Words: Esophageal atresia, Tracheoesophageal fistula, Premature infant

\section{INTRODUCTION}

Esophageal atresia (EA) is the most common congenital esophageal anomaly ${ }^{1)}$. However, EA with a proximal tracheoesophageal fistula (TEF; gross type B) occurs in only $1 \%$ to $2 \%$ of cases. The presence of TEF differentiates type B EA from type A EA. However, because both types often involve long-gap EA, surgery is difficult and the prognosis is poor owing to many postoperative complications. Moreover, there are yet no established surgical methods.
Received: 14 July 2018

Revised: 22 August 2018

Accepted: 8 September 2018

Correspondence to: Yong-Hoon Cho

Division of Pediatric Surgery, Department of Surgery, Pusan National University Yangsan Hospital, Pusan National University School of Medicine, 20 Geumo-ro, Mulgeum-eup, Yangsan 50612, Korea

Tel: +82-55-360-2124

Fax: +82-55-360-2154

E-mail: choyh70@pusan.ac.kr

Copyright(c)

By Korean Society of Neonatology.

All right reserved.

This is an Open-Access article distributed under the terms of the Creative Commons Attribution Non-Commercial License (http://creativecommons.org/licenses/ by-nc/4.0), which permits unrestricted non-commercial use, distribution, and reproduction in any medium, provided the original work is properly cited. 
In particular, low birth weight, congenital cardiac defects, and compromised respiratory function in conjunction with EA are known risk factors for a poor prognosis ${ }^{2-4)}$.

Among the studies published in Korea, a clinical analysis by Chang et al. ${ }^{5)}$ with a relatively high number of patients with EA did not include any patients with type B EA, indicating the rarity of this type. Among the case reports on long-gap EA in Korea, two reported on type $\mathrm{A} \mathrm{EA}^{6,7)}$ but only one reported a case of type $\mathrm{B}$ $\mathrm{EA}^{8)}$. In the latter case, the surgical method used was reverse gastric tube interposition, which is different from our case. We encountered the rare defect of type B EA in a male infant, and report here on the treatment process, from the initial misdiagnosis to the performance of esophageal reconstruction with gastric pull-up. We believe that this report can serve as a basis for treating similar patients in the future.

\section{CASE REPORT}

Written informed consent was obtained from the infant's parents for the publication of this case.

A male infant with a birth weight of $1,616 \mathrm{~g}$ was born at 35 weeks and 5 days of gestation via a cesarean section. Intubation was performed owing to poor initial crying and activity, desa- turation, and moaning. The patient was transferred to Pusan National University Children's Hospital with a suspicion of EA based on failed gastric tube insertion.

The 34-year-old mother had a history of three miscarriages, and this infant was her first live delivery. The infant's birth weight $(1,616 \mathrm{~g})$, height $(41 \mathrm{~cm})$, and head circumference $(30 \mathrm{~cm})$ were all below the 10th percentile for gestational age. On the basis of the detection of a twisted orogastric tube at the T2 level, absence of stomach gas, and relatively clear lungs on serial infantography, a diagnosis of pure EA (gross type A) was made (Figure 1A). A small-caliber sum-up tube remained in the upper pouch and was suctioned continuously.

A gastrostomy tube was placed, and a "feed and grow" approach was implemented in the neonatal intensive care unit at 2 days after birth. Total corrective surgery was planned when the patient's body weight reached $5 \mathrm{~kg}$. Although weaning from mechanical ventilation was possible at 5 days after birth, endotracheal intubation and mechanical ventilator care were needed because of subsequent recurrent atelectasis and dyspnea (Figure 1B). On chest computed tomography performed at 42 days after birth for respiratory assessment, although no fistula was found, the ends of the esophagus were confirmed at the T2 and T9 levels, indicating EA associated with a long gap involving seven vertebrae. However, because TEF was found on bronchoscopy

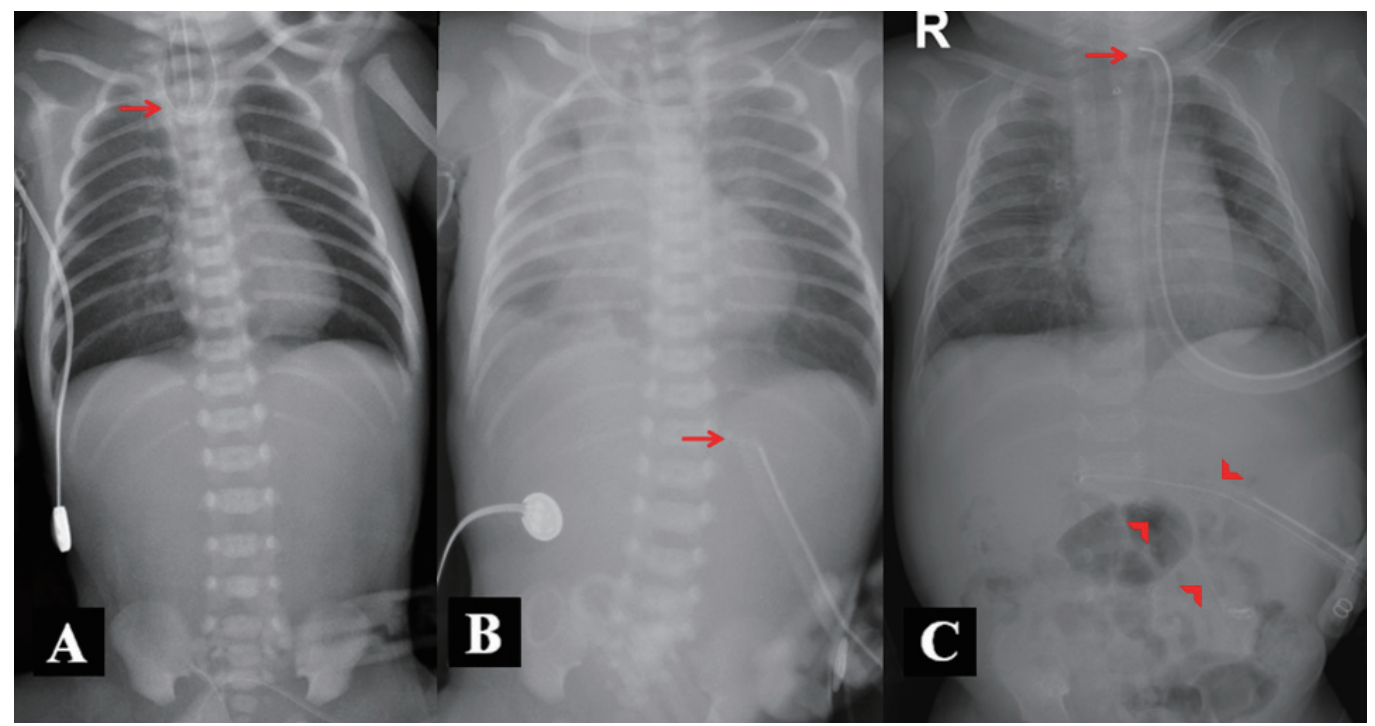

Figure 1. (A) Day 1 after birth: infantography of the patient with suspected gross type A esophageal atresia demonstrating the curling of a radiopaque Levin tube in the upper pouch (arrow) and the absence of luminal gas below the diaphragm. The absence of stomach gas was continuous on serial infantography. (B) Day 48 after birth: increased haziness in the right lung due to recurrent pneumonia. The arrow indicates a feeding tube inserted via gastrostomy. (C) Day 65 after birth: improved lung condition after tracheoesophageal fistula excision and cervical end esophagostomy (arrow). A Levin tube was inserted into the jejunum for continuous feeding (arrowheads). 
performed at 48 days after birth, the diagnosis was changed to type B EA and weaning from mechanical ventilation became possible after excision of the TEF and cervical end esophagostomy.

At 49 days after birth (weight $2.5 \mathrm{~kg}$ ), leakage of milk (more than half of the consumed volume) into the gastrostomy site occurred, causing skin damage. Diagnostic imaging with contrast infusion through the gastrostomy site confirmed a $30-\mathrm{mL}$ stomach volume, and a Levin tube was advanced into the jejunum through the gastrostomy to establish continuous feeding (Figure 1C). We determined that the stomach with a small volume of $30 \mathrm{~mL}$ was suitable as a replacement for the esophagus, and at 141 days after birth when his body weight had reached $4.7 \mathrm{~kg}$, esophageal reconstruction with gastric pull-up was performed. Esophagography was performed on postoperative day 7 to ensure the absence of leakage (Figure 2A). He was discharged 21 days postoperatively after confirming the absence of acid reflux on upper gastrointestinal endoscopy (Figure 2B). On assessment for associated vertebral defects, anal atresia, cardiac defects, TEF, renal anomalies, and limb abnormalities (VACTERL), we also identified an atrial septal defect. The toxoplasmosis, rubella, cytomegalovirus, herpes simplex, and others (TORCH) screening for congenital infections tested negative, and the patient had normal hearing test, neonatal screening test, and cranial ultrasound results.

Currently, the patient is 12 months old but his weight $(6.5 \mathrm{~kg})$, height $(64.1 \mathrm{~cm})$, and head circumference $(43.8 \mathrm{~cm})$ are still below the 10th percentile for corrected age. Although catch-up growth did not occur, he has been showing appropriate development for his age. His diet consists mostly of special anti-reflux formula, although he is currently eating baby food, three times a day, without any complications such as anastomotic stricture or aspiration pneumonia. His growth, development, and long-term complications will be monitored.

\section{DISCUSSION}

EA, which can be life threatening without corrective surgery, is an esophageal malformation occurring in approximately 1 in 3,500 live births ${ }^{1,9)}$. Most cases involve pulmonary complications, and effective coordination and collaborative care among specialists are required to successfully treat cases of EA accompanied by congenital anomalies such as VACTERL ${ }^{10)}$. Specifically, a careful therapeutic approach is essential for treating EA of gross types A or B because of the long gap between the upper and lower esophageal pouches, which complicates the surgical procedure $^{11)}$.

In the current case, a fistula was initially absent on a chest computed tomography scan but was detected during bronchoscopy performed for recurrent pneumonia. The course of events resulted in a diagnosis of gross type B EA and a revised treatment plan. Although chest radiography is generally adequate to diagnose EA, the EA types are categorized according to the presence

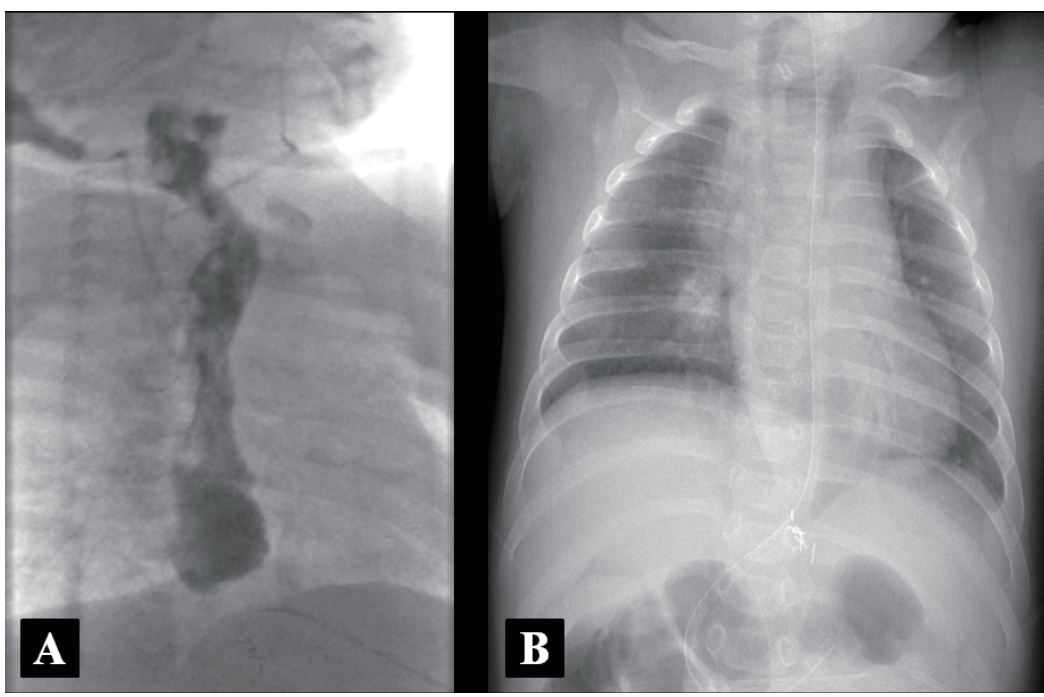

Figure 2. (A) Esophagogram taken 7 days after esophageal reconstruction with gastric pull-up, showing no leakage or stricture. (B) Chest radiograph taken 30 days after esophageal reconstruction with gastric pull-up. 
or absence and the location of a TEF. To determine the surgical method for treating long-gap EA, accurate gap measurement and identification of a fistula are crucial. In an international survey ${ }^{12)}$, preoperative bronchoscopy was found to have been performed in only $43 \%$ of cases. Atzori et al. ${ }^{13)}$ reported that preoperative bronchoscopy was beneficial in $45.2 \%$ of patients, and the planned surgical method was revised in $24.2 \%$ of cases, highlighting the importance of preoperative bronchoscopy. Accurate identification of the anatomical structure in a patient with EA is essential for selecting the appropriate surgical method and for optimal timing of treatment. Impaired lung function, indicated by the presence of pneumonia or need for a ventilator, is a poor prognostic factor ${ }^{14,15)}$. Preoperative bronchoscopy is recommended, given its advantageous effects in terms of patient survival, length of hospitalization, and cost.

In general, growth is limited in patients with intrauterine growth retardation $^{16)}$. To promote growth preoperatively in our patient, we initiated aggressive parenteral nutrition and simultaneous feeding. The goal was early termination of parenteral nutrition to prevent infections via the central line, and enteral nutrition was selected to strengthen the immune system and prevent the atrophy of intestinal cells ${ }^{17)}$. Our patient's stomach volume was insufficient for bolus feeding for total enteral nutrition after a body weight of $2.5 \mathrm{~kg}$ was reached. Consequently, a tube was inserted into the jejunum via gastrostomy to achieve continuous enteral nutrition, which facilitated growth and eventually allowed surgical treatment. Although the long-term application of continuous tube feeding is not recommended, considering the biological aspects of feeding-associated insulin secretion, rendering it a less-than-optimal method of feeding, we believe that its short-term application is a more effective method than total parenteral nutrition.

Although patients with type B EA typically have long-gap EA and can be treated using various surgical methods, including staged repair and esophageal replacement, guidelines have not been established. Historically, immediate or delayed primary repair has been considered inappropriate for long-gap EA, and long-gap EA is an absolute indication of esophageal substitution. However, in the last 20 years, there has been a dramatic increase in the number of cases of long-gap EA with successful esophageal repair $^{18,19)}$. As our patient had a low birth weight and a compromised respiratory condition, delayed surgery was planned. Although an insufficient stomach volume was confirmed on the mid-treatment assessment, resulting in continuous tube feeding via a gastrojejunal tube, esophageal reconstruction with gastric pull-up was selected based on this finding.

Endoscopic surveillance is recommended to confirm ulcerative esophagitis and dysplastic epithelial changes in the esophageal remnants. In the present case, the absence of severe gastroesophageal reflux was determined with postoperative endoscopy. Although there have been reports of rare cases accompanied by obstructive intestinal disease ${ }^{20)}$, our patient did not have any other intestinal disease and oral feeding without dysphagia was possible. Although the initial failure to detect a fistula likely exacerbated the condition of the lungs, leading to conventional ventilator care for 51 days preoperatively and postoperatively and oxygen treatment for 93 days, the patient was discharged without home oxygen.

We identified a limitation in the treatment of our patient in that the fistula was found after the condition of the lungs had deteriorated, although the diagnosis and treatment plan were revised upon fistula detection on preoperative bronchoscopy. Patients suspected of having pure EA require a thorough examination for subtle tracheoesophageal connections to prevent lung complications. As our patient had a small stomach volume, esophageal reconstruction with gastric pull-up was performed successfully after continuous feeding through a gastrojejunal tube, and oral feeding was ultimately possible. Feeding to promote growth before total corrective surgery must be tailored to the individual patient's needs.

Long-term follow-up observations should be conducted to further confirm our findings in this patient; however, we conclude that esophageal reconstruction with gastric pull-up is a suitable surgical method for patients with a small stomach volume. Furthermore, continuous collaboration between neonatologists and pediatric surgeons is crucial for a successful treatment of premature infants with fetal growth retardation with type B EA.

\section{CONFLICT OF INTEREST}

No potential conflict of interest relevant to this article was reported. 


\section{ACKNOWLEDGMENTS}

We greatly acknowledge the cooperation of the medical staff of Pusan National University Children's Hospital, including the neonatal intensive care unit team, pediatric surgeons, radiologists, cardiologists, pulmonologists, and gastroenterologists.

\section{REFERENCES}

1. Spitz L. Oesophageal atresia. Orphanet J Rare Dis 2007;2:24.

2. Spitz L, Kiely EM, Morecroft JA, Drake DP. Oesophageal atresia: at-risk groups for the 1990s. J Pediatr Surg 1994;29:723-5.

3. Yagyu M, Gitter H, Richter B, Booss D. Esophageal atresia in Bremen, Germany: evaluation of preoperative risk classification in esophageal atresia. J Pediatr Surg 2000;35:584-7.

4. Sinha CK, Haider N, Marri RR, Rajimwale A, Fisher R, Nour S. Modified prognostic criteria for oesophageal atresia and tracheo-oesophageal fistula. Eur J Pediatr Surg 2007;17:153-7.

5. Chang EY, Chang HK, Han SJ, Choi SH, Hwang EH, Oh JT. Clinical characteristics and treatment of esophageal atresia: a single institutional experience. J Korean Surg Soc 2012;83:43-9.

6. Cho SH, Oh BS, Lee DJ. End to end anastomosis of type A (long gap) esophageal atresia in 1200 gram premature baby: a case report. Korean J Thorac Cardiovasc Surg 1997;30:236-40.

7. Lee DS, Namgung H, Yoon JS. Long gap esophageal atresia successfully treated by esophageal lengthening using external traction sutures. J Korean Assoc Pediatr Surg 2005;11:165-70.

8. Kim HT, Bae WJ, Jung SL, Lee JW. A case of the esophageal atresia with proximal tracheoesophageal fistula. J Korean Pediatr Soc 1998;41:861-4.

9. Torfs CP, Curry CJ, Bateson TF. Population-based study of tracheoesophageal fistula and esophageal atresia. Teratology 1995;52:220-32.
10. Esophageal atresia and tracheoesophageal fistula. In: Martin RJ, Faranoff AA, Walsh MC, editors. Fanaroff and Martin's neonatal-perinatal medicine: diseases of the fetus and infant. 10th ed. Philadelphia: Elsevier Saunders, 2015:1395-400.

11. Koivusalo AI, Sistonen SJ, Lindahl HG, Rintala RJ, Pakarinen MP. Long-term outcomes of oesophageal atresia without or with proximal tracheooesophageal fistula: gross types A and B. J Pediatr Surg 2017;52:1571-5.

12. Zani A, Eaton S, Hoellwarth ME, Puri P, Tovar J, Fasching G, et al. International survey on the management of esophageal atresia. Eur J Pediatr Surg 2014;24:3-8.

13. Atzori P, Iacobelli BD, Bottero S, Spirydakis J, Laviani R, Trucchi A, et al. Preoperative tracheobronchoscopy in newborns with esophageal atresia: does it matter? J Pediatr Surg 2006;41:10547.

14. Waterston DJ, Carter RE, Aberdeen E. Oesophageal atresia: tracheo-oesophageal fistula. A study of survival in 218 infants. Lancet 1962;1:819-22.

15. Poenaru D, Laberge JM, Neilson IR, Guttman FM. A new prognostic classification for esophageal atresia. Surgery 1993; 113:426-32.

16. Botero D, Lifshitz F. Intrauterine growth retardation and longterm effects on growth. Curr Opin Pediatr 1999;11:340-7.

17. Neu J. The human gut microbiota in perinatology and neonatology. Semin Fetal Neonatal Med 2016;21:367.

18. Boyle EM Jr, Irwin ED, Foker JE. Primary repair of ultra-long-gap esophageal atresia: results without a lengthening procedure. Ann Thorac Surg 1994;57:576-9.

19. Foker JE, Linden BC, Boyle EM Jr, Marquardt C. Development of a true primary repair for the full spectrum of esophageal atresia. Ann Surg 1997;226:533-41.

20. Jung SL. A case of the esophageal atresia with distal tracheoesophageal fistula associated with duodenal obstrction. J Korean Soc Neonatol 1998;5:217-20. 